\title{
Direct Optical Visualization of Graphene and Its Nanoscale Defects on Transparent Substrates
}

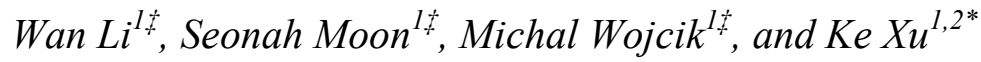

${ }^{1}$ Department of Chemistry, University of California, Berkeley, California 94720, USA

${ }^{2}$ Division of Molecular Biophysics and Integrated Bioimaging, Lawrence Berkeley National Laboratory, Berkeley, California 94720, USA

*Correspondence to: xuk@berkeley.edu

\section{Supporting Information}

\section{Materials and Methods}

\section{Theory}

Our derivations and notations follow conventions of thin-film optics. ${ }^{1}$ Layer configuration of the system is given in Fig. 1a. Incident light of wavelength $\lambda$ and intensity $I_{\mathrm{I}}$ enters the sample from the substrate side, successively encounters graphene and top medium, and leaves as reflected $(R)$ and transmitted $(T)$ light. The refractive indices of the substrate, graphene, and top medium are respectively denoted as $n_{1}, n_{2}$, and $n_{3}$. Graphene thickness is $d_{2}$.

The Fresnel coefficients at the substrate-graphene and graphene-top medium interfaces are respectively $r_{12}=\frac{n_{1}-n_{2}}{n_{1}+n_{2}}, t_{12}=\frac{2 n_{1}}{n_{1}+n_{2}}$ and $r_{23}=\frac{n_{2}-n_{3}}{n_{2}+n_{3}}, t_{23}=\frac{2 n_{2}}{n_{2}+n_{3}}$.

The transfer matrices at the two interfaces are $T^{12}=\frac{1}{t_{12}}\left[\begin{array}{cc}1 & r_{12} \\ r_{12} & 1\end{array}\right]$ and $T^{23}=\frac{1}{t_{23}}\left[\begin{array}{cc}1 & r_{23} \\ r_{23} & 1\end{array}\right]$.

The transfer matrix in graphene is $T^{2}=\left[\begin{array}{cc}e^{i \varphi} & 0 \\ 0 & e^{-i \varphi}\end{array}\right]$, where $\varphi=\frac{2 \pi}{\lambda} n_{2} d_{2}$.

The transfer matrix for the system is:

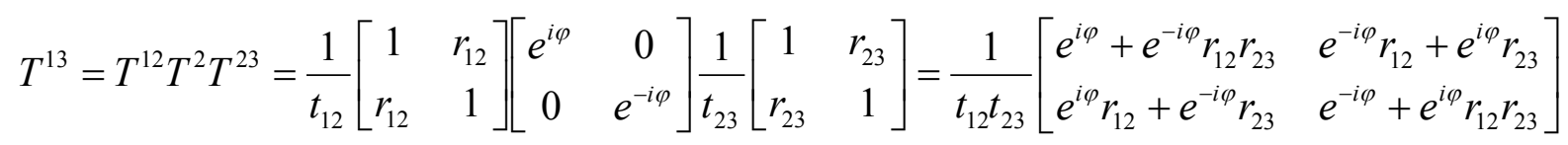

The reflection coefficient is:

$r=\frac{T_{21}^{13}}{T_{11}^{13}}=\frac{e^{i \varphi} r_{12}+e^{-i \varphi} r_{23}}{e^{i \varphi}+e^{-i \varphi} r_{12} r_{23}}$ 
Intensity of reflected light, which is recorded experimentally in IRM images, is thus:

$I=|r|^{2} I_{I}=\left|\frac{e^{i \varphi} r_{12}+e^{-i \varphi} r_{23}}{e^{i \varphi}+e^{-i \varphi} r_{12} r_{23}}\right|^{2} I_{I}$

Meanwhile, intensity without graphene (a direct $n_{1}-n_{3}$ interface) is: $I_{0}=\left|\frac{n_{1}-n_{3}}{n_{1}+n_{3}}\right|^{2} I_{\mathrm{I}}$.

It can be shown that this result is equal to that obtained through Eqn (1) for $d_{2}=0$ (number of graphene layer, $m=0$ ).

\section{Preparation of graphene on different substrates}

\section{a. Wet transfer with PMMA protection}

Graphene on glass, quartz, $\mathrm{CaF}_{2}$ and Aclar (polychlorotrifluoroethene) substrates were prepared through the standard wet-transfer method with PMMA protection. ${ }^{2,3} \mathrm{CVD}$ graphene on copper foils (Graphene Supermarket, Calverton, NY) was spin-coated with a $\sim 150 \mathrm{~nm}$ layer of polymethyl methacrylate (PMMA 495 A4, MicroChem, Newton, MA). After the copper was removed in an etching solution $\left(5 \% \mathrm{HCl}+20 \% \mathrm{FeCl}_{3}\right)$, the graphene-PMMA stack was transferred to a fresh water bath so it floated on the water surface. Water bath transfer was repeated three times to remove traces of ferric chloride. The PMMA-protected graphene film was then transferred to the target substrates. PMMA was removed in two steps using anisole (15 $\mathrm{min}$ ) and acetone (1-2 hours) followed by a rinse in isopropanol (10 $\mathrm{min})$, and the sample was dried with nitrogen gas.

\section{b. Wet transfer without PMMA protection:}

CVD graphene on copper was floated on top of an etching solution for 5-10 minutes to remove copper. As soon as the copper layer became invisible, a cleaned polymer substrate was used to carefully stamp the graphene piece from the top. The polymer substrate with graphene was air-dried for $\sim 20$ minutes and then rinsed with DI water.

\section{c. Dry transfer using thermal release tape}

Graphene transfer tape (Graphene Supermarket, GTT-5pk) was applied to a piece of CVD graphene on copper and pressed thoroughly. Copper was removed in an etching solution for $\sim 10$ minutes. Tape with graphene was rinsed in fresh DI water for three times and then briefly air-dried. The tape was applied to the polymer substrate, pressed and scraped thoroughly. A hotplate was used to heat the sample to $\sim 90^{\circ} \mathrm{C}$ for release of the tape.

\section{d. Dry transfer with $3 M$ scotch tape}

3M Scotch 105 Magic Tape was applied to a piece of CVD graphene on copper and pressed and scraped thoroughly. Copper was removed in an etching solution for $\sim 10$ minutes. Sample was rinsed with fresh DI water for three times. After brief air-drying, the tape with 
graphene was applied on a cleaned polymer substrate, pressed and scraped thoroughly. The tape was gently taken off from the polymer substrate.

\section{e. Nano-patterning of graphene}

Defined nano-patterns of graphene (Fig. 2a) were fabricated using focused ion beam (FIB). A gallium ion beam in a FEI Quanta SEM/FIB system was used to pattern CVD graphene on a copper foil, and the patterned graphene was transferred to a glass substrate using PMMA-protected wet transfer as described above. Mechanically disrupted graphene samples (e.g., Fig. 2bc) were produced by immersing the samples in acetone and using a pipette to generate air bubbles at the graphene surface, a process that emulates what is often encountered in the lift-off process of photolithography for device nanofabrication.

\section{Interference reflection microscopy (IRM)}

IRM was performed on a conventional Olympus IX73 inverted wide-field epifluorescence microscope that was equipped with a standard lamp for fluorescence microscopy (U-HGLGPS). The fluorescence filter cube was configured with a 50/50 beam splitter (Chroma 21000) and a 530/10 nm band pass filter (Chroma D532/10x) as the excitation filter. While the use of a band pass filter facilitated comparison of results with theory, the obtained contrast was relatively insensitive to the wavelength, and comparable contrast may be obtained without using an optical filter (Fig. S2). No emission filter was used. Objective lenses were an Olympus UplanFl 100× oil-immersion objective (NA 0.9 with iris diaphragm) and an Olympus UplanSapo 60× water-immersion objective (NA 1.2). Significantly reduced contrast was observed when dry objective lens was employed due to back-reflections at air-glass interfaces ( $\sim 8 \%$ for monolayer graphene on glass when using an UplanSapo $20 \times$ objective), and so is not recommended. IRM images were acquired at 16-bit bit-depth using an Andor Zyla 4.2 sCMOS camera at $1024 \times 1024$ pixels with $20 \mathrm{~ms}$ integration time ( $\sim 50$ frames per second) or at $512 \times 512$ pixels to achieve a $10 \mathrm{~ms}$ integration time (100 frames per second or $4 \times$ video-rate). Effective pixel size, $l_{\text {pixel }}$, was $65 \mathrm{~nm}$ and 108 $\mathrm{nm}$ when using the $100 \times$ and $60 \times$ objectives, respectively. The microscope field diaphragm was closed down to slightly smaller than the $1024 \times 1024$ frame size to reject stray light from oblique angles, hence the black edges in images. Comparable results were obtained when the entire sample was immersed in water, or when a droplet of water was deposited at the area of observation. Eqn (1) predicts that the final results only depend on the index of refraction, rather than the nature, of the top medium. In this work we focused on water as it is most accessible and uniquely compatible with all the substrates used in this study.

\section{SEM and AFM characterizations}

The conductivity of graphene itself was utilized to enable SEM characterization of graphene on the insulating substrates. ${ }^{4}$ The sample was mounted on a standard metallic sample mount using carbon tape, and a small amount of silver colloid paint (Ted Pella 16031) was used to create a conductive bridge between graphene and the sample mount. SEM imaging was performed on a FEI Quanta 3D FEG system in secondary-electron mode. 
Comparison of images obtained at different acceleration voltages indicated that best contrast was obtained at $2 \mathrm{kV}$ (Fig. S3), which was selected for comparison with IRM results.

AFM images were taken on an Asylum MFP-3D system in tapping mode using aluminum-coated probes (Tap150Al-G; BudgetSensors). Nominal values of the force constant, resonance frequency, and tip radius were $5 \mathrm{~N} / \mathrm{m}, 150 \mathrm{kHz}$, and $<10 \mathrm{~nm}$, respectively. AFM data were processed using WsXM. ${ }^{5}$

\section{Concurrent IRM and electrical characterization of graphene subject to uniaxial stretching}

\section{a. Sample preparation}

For stretching experiments, CVD graphene $(\sim 10 \times 4 \mathrm{~mm})$ was deposited at the center of a $\sim 60 \times 6 \mathrm{~mm}$ strip of a $0.2 \mathrm{~mm}$-thick Aclar 33C film (Ted Pella, Redding, CA). A liquid metal, GaInSn (68.5\%:21.5\%:10\%), was employed as contact electrodes to ensure reliable contact to graphene during stretching (Scheme S1 inset). Two thin copper wires were first connected to graphene using silver paint and glued down onto the substrate with epoxy. GaInSn liquid metal was then applied to connect the graphene surface with the copper wires; final distance between electrodes was $\sim 8 \mathrm{~mm}$. The liquid metal wetted the graphene surface well and so maintained highly stable junction conductance during stretching. A control sample in which GaInSn liquid metal directly bridged two copper wires on the Aclar substrate showed a highly stable resistance of 1.36-1.38 $\Omega$ during a similar stretching process, which is more than three orders of magnitude smaller than the measured resistance of graphene.

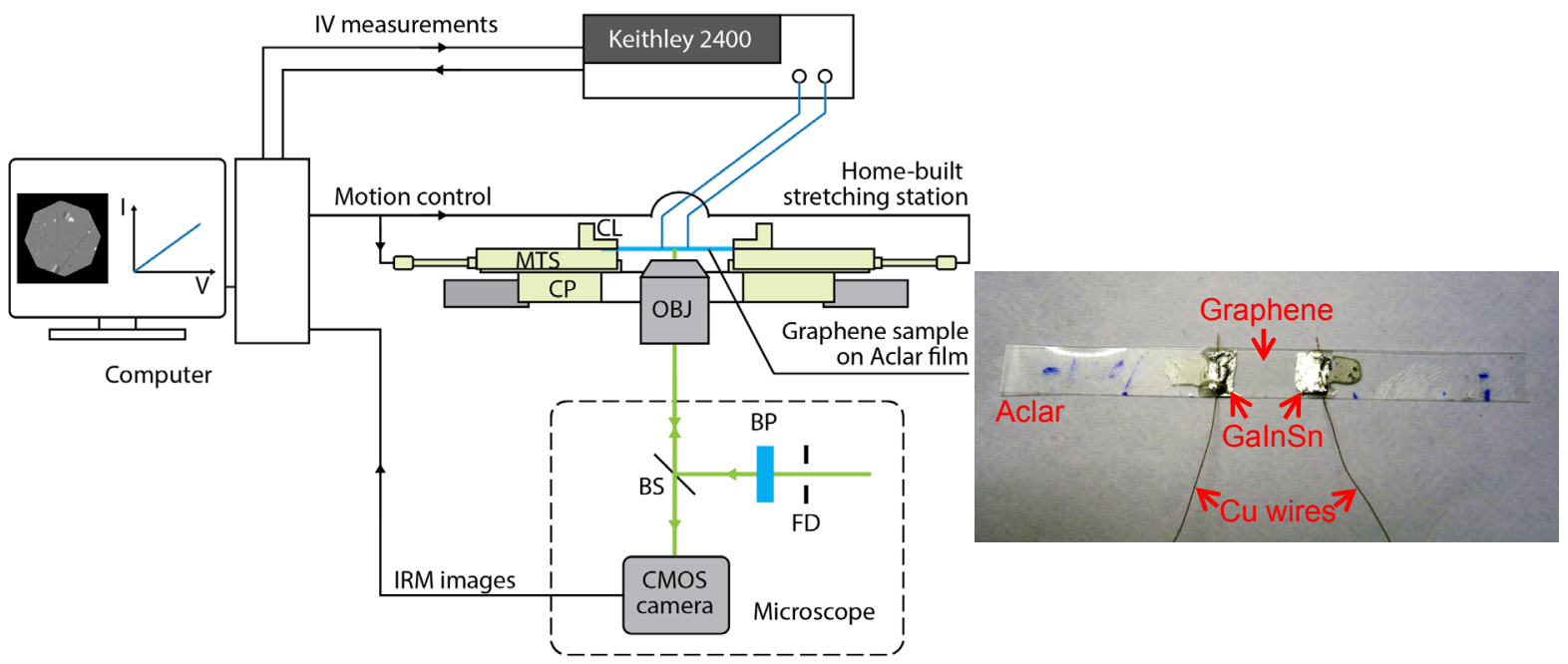

Scheme S1. Experimental setup for concurrent IRM and electrical characterization of graphene under stretching. CP: Central plate of the microscope. MTS: motorized translation stage. CL: clamp. Inset: photo of the sample. A GalnSn liquid metal was employed to achieve reliable contact to graphene during stretching.

\section{b. Measurement system}


The measurement system (Scheme S1) was comprised of three sub units, namely a homebuilt tensile-testing station to apply uniaxial strain, IRM to monitor in situ structural changes in graphene under strain, and a Keithley 2400 SourceMeter to concurrently monitor the electrical properties of graphene.

The tensile-testing station was constructed by mounting two single-axis motorized translation stages (PT1-Z8, Thorlabs) onto the central plate of the microscope stage, face to face with a $50 \mathrm{~mm}$ gap at zero displacement. The strip-shaped sample was clamped at the two ends to the two stages so that its long axis is aligned with the translational axes of both stages. A computer program was developed to simultaneously displace the two motorized stages to opposite directions at the same rate. The system is effective for stretching graphene up to a strain of $\sim 9.5 \%$, when the Aclar film starts to yield. Strain was measured by examining the actual displacements of structural features within the sample from the obtained IRM images.

IRM system was as described above, but with the addition of an extension tube for the objective lens to account for the increase in height due to the stretching stages. To calculate the width of nano-cracks in monolayer graphene, which are often smaller than the diffraction-limited resolution of optical microscopy $(\sim 300 \mathrm{~nm})$, we integrated by pixel, across the crack, the intensity difference when compared to continuous graphene, $\Sigma \Delta I_{\text {crack }}$. The crack width was then determined by $w=l_{\text {pixel }} \Sigma \Delta I_{\text {crack }} /\left(I_{0}-I_{1}\right)$. Here $l_{\text {pixel }}$ is the effective pixel size, and $I_{0}$ and $I_{1}$ are the experimentally measured light intensity per pixel on blank substrate and on continuous monolayer graphene, respectively. Crack density is calculated as the number of nano-cracks per unit length in the stretching direction, averaged across the image.

A Keithley 2400 SourceMeter was used to monitor the electrical properties of graphene during stretching. Current through graphene was recorded as the voltage was continuously swept in loops between $-12 \mathrm{mV}$ and $12 \mathrm{mV}$. Resistance was determined by fitting to the resultant, highly linear I-V data. 

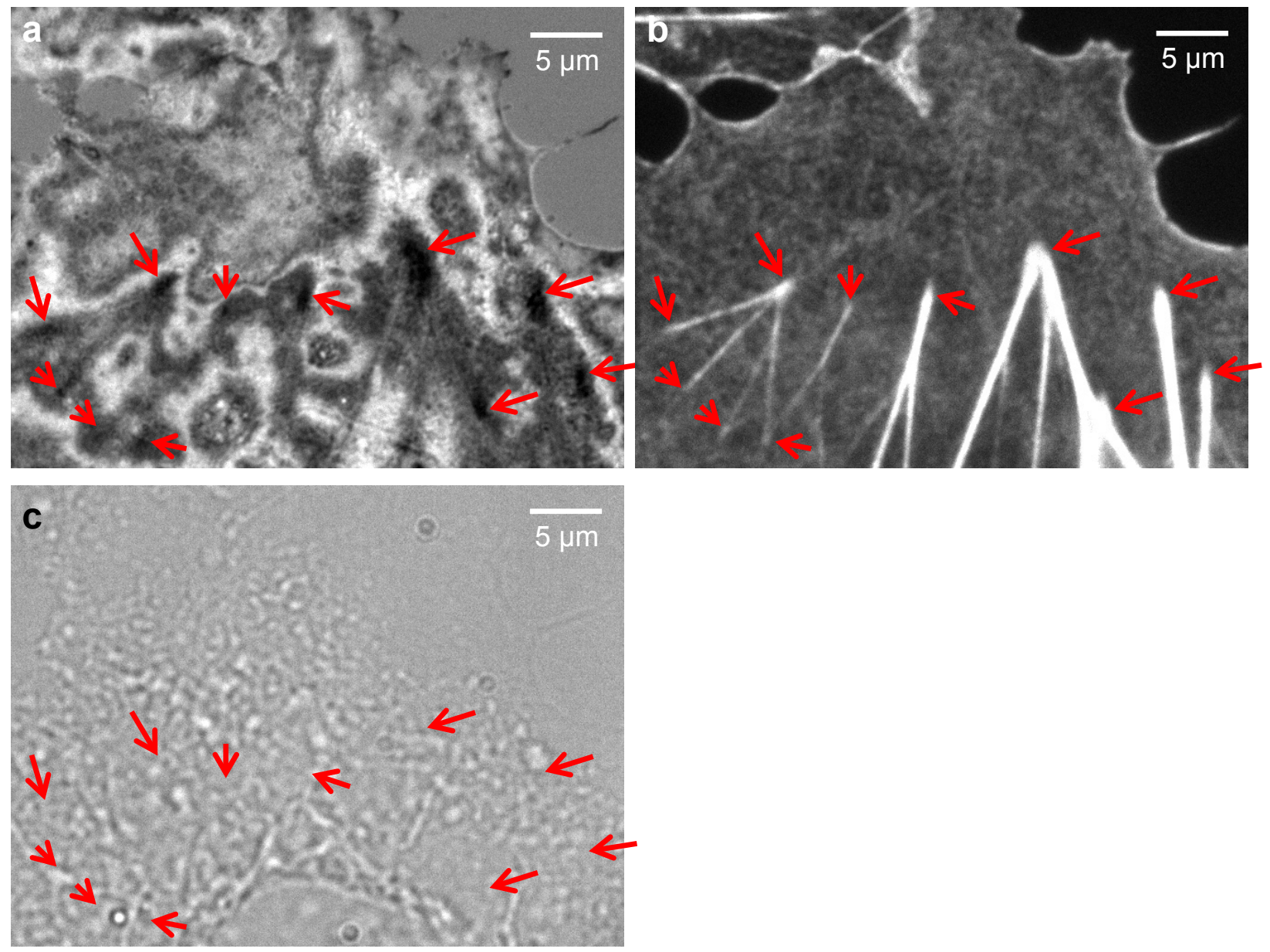

Figure S1. In cell biology, IRM provides useful contrast for cell adhesion sites. (a) IRM image of COS-7 fibroblast cells taken under the same condition as graphene in this study. (b) Fluorescence microscopy of the phalloidin-labeled actin cytoskeleton of the same area. (c) Bright-field transmission light microscopy of the same area. Arrows in (a) point to dark areas in IRM that correspond to cell adhesion sites, ${ }^{6-8}$ which match well to focal adhesion sites at the ends of actin stress fibers ${ }^{9,10}$ as visualized in the fluorescence image (b). In comparison, no contrast for adhesion sites is observed in transmission microscopy (c). 

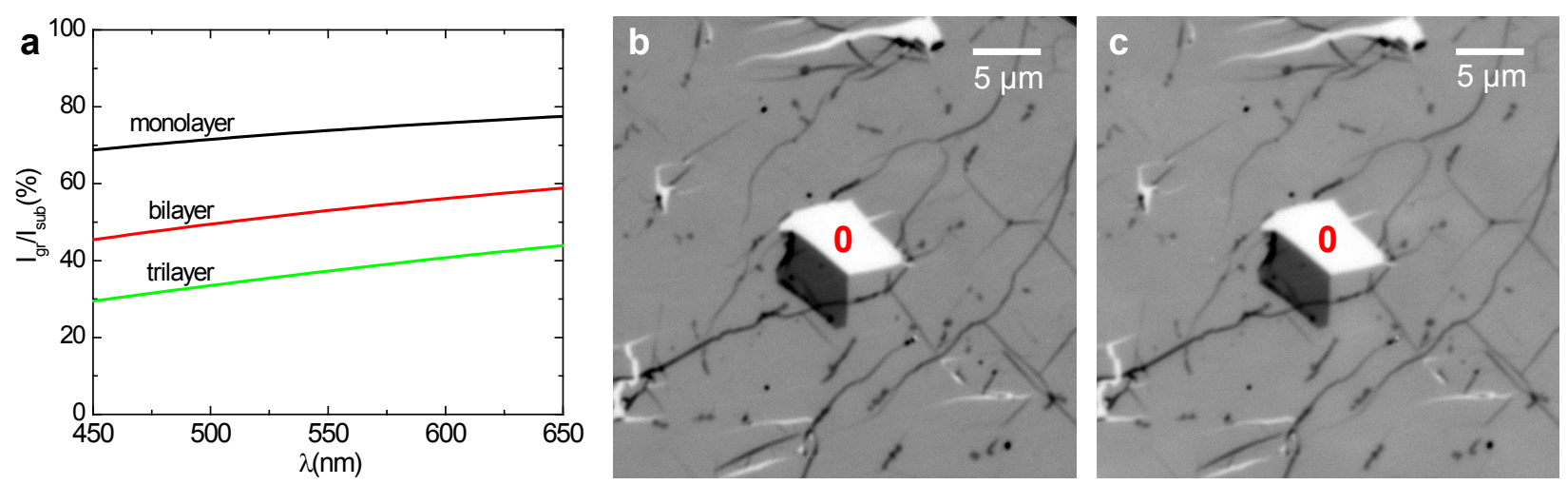

Figure S2. IRM contrast of graphene is relatively insensitive to wavelength. (a) Theoretical IRM signal (normalized by signal intensity on bare substrate without graphene) for graphene of different layers on glass, as a function of wavelength according to Eqn. 1. (b) Experimental result with the 530/10 nm band pass filter (condition for all results presented in this work). " 0 " marks an area with no graphene coverage. (c) Experimental result for the same sample without the use of any optical filters: here the recorded signal is the weighted average of all wavelengths in the spectrum of the lamp convolved with the wavelength-dependent sensitivity of the camera. In our setup the resultant contrast ( $30 \%$ for monolayer graphene) happens to be comparable to that when the $532 / 10 \mathrm{~nm}$ bandpass filter is used. 


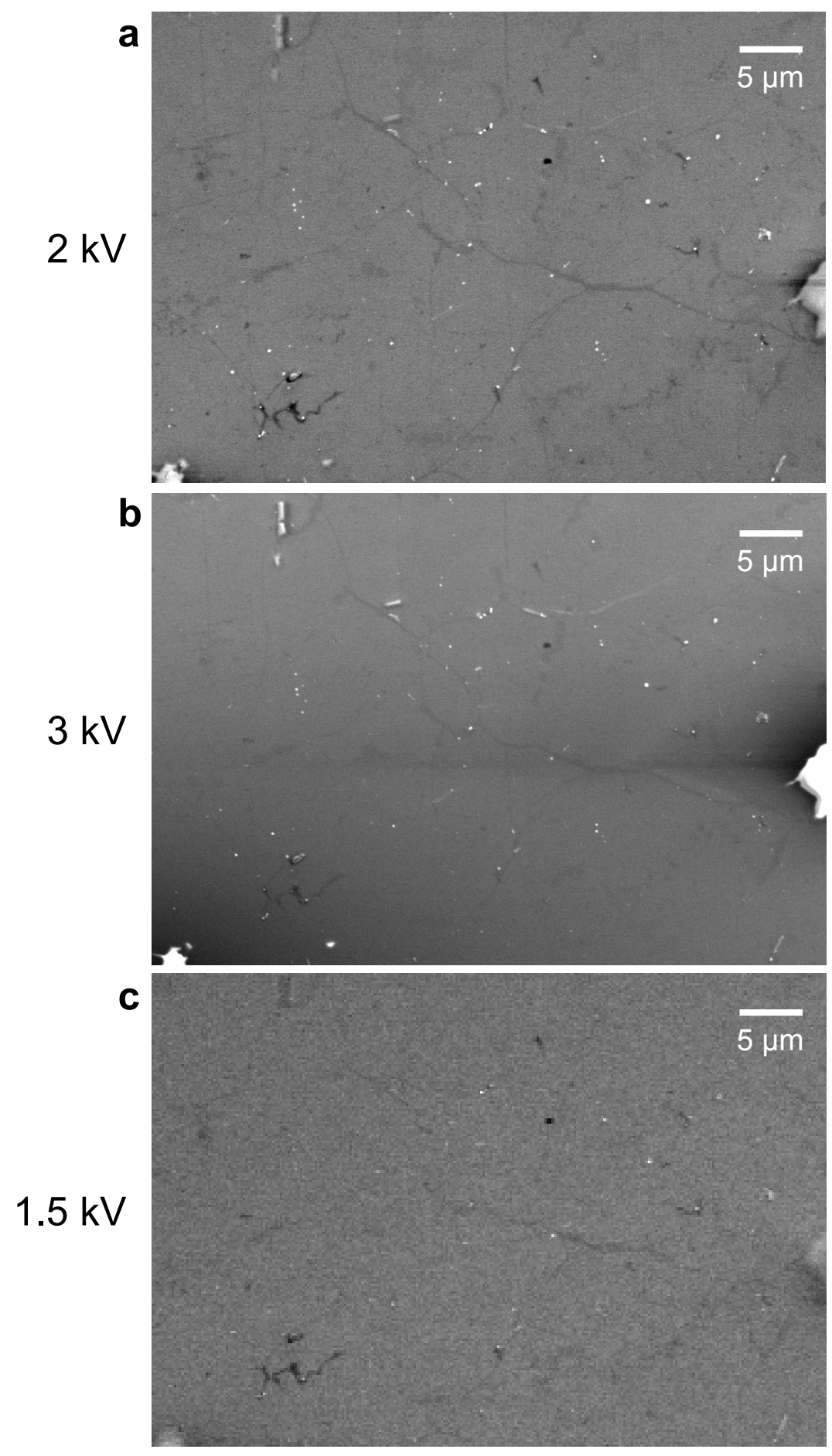

Figure S3. SEM of graphene on glass at different accelerate voltages ( $\left.\boldsymbol{V}_{\mathbf{0}}\right)$. (a) $V_{0}=2 \mathrm{kV}$, corresponding to Fig. 2e: this provides the best contrast. (b) $V_{0}=3 \mathrm{kV}$ : Stronger charging effect is observed at the exposed glass surface, and lower contrast is observed for wrinkles. (c) $V_{0}=1.5$ $\mathrm{kV}$ : Surface charging is reduced, but image contrast and resolution both dropped. Taken together, these results illustrate the difficulties of using SEM to characterize graphene on the fully insulating glass substrate. 

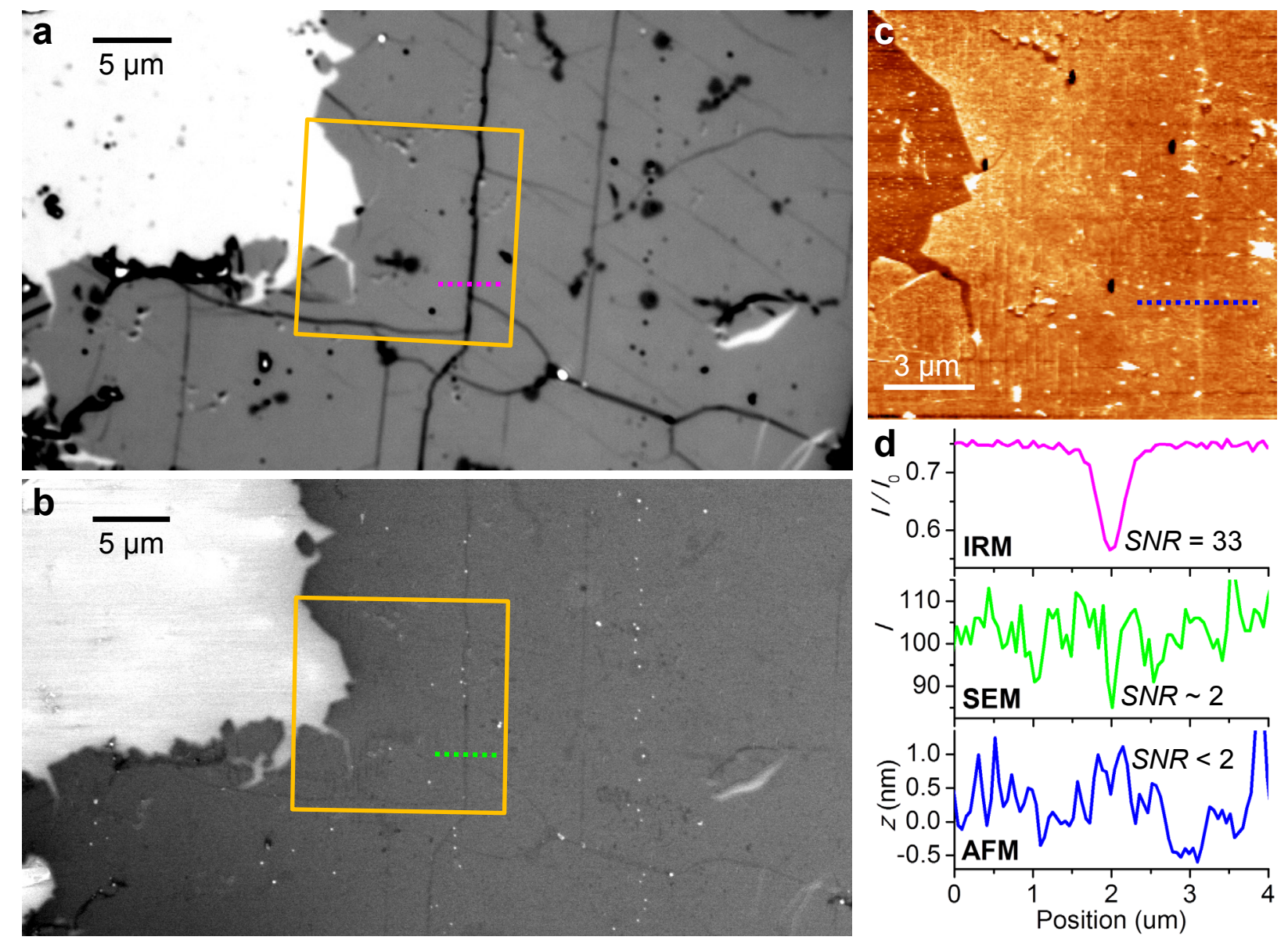

Figure S4. Additional correlated results of IRM (a), SEM (b), and AFM (c). AFM image in (c) corresponds to orange boxes in $(a, b)$. (d) Intensity and height profiles across the same major wrinkle, corresponding to the dotted lines in the three imaging modes. 

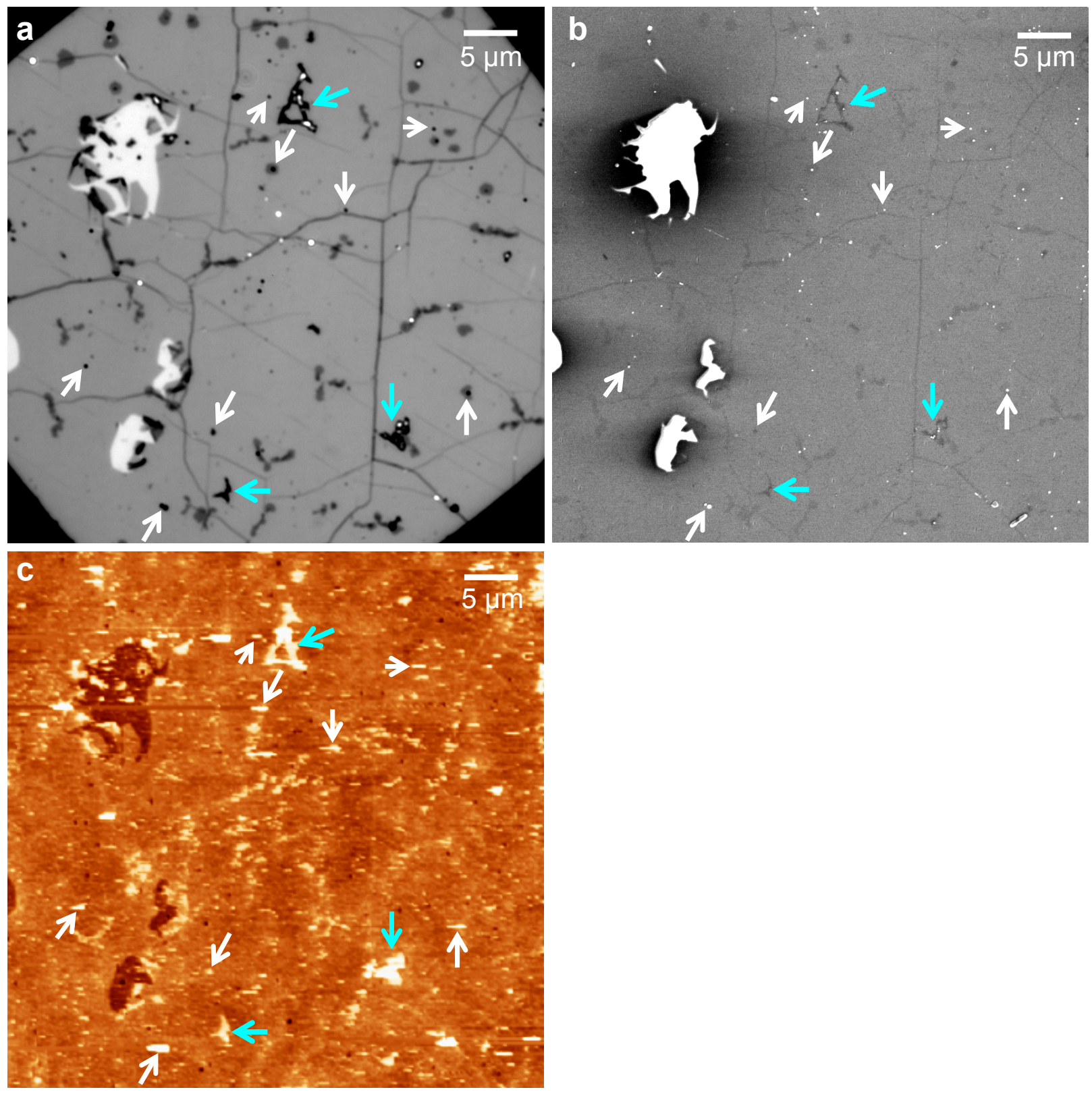

Figure S5. Visualization of nanoscale contaminants. (a-c) Correlated results of IRM (a), SEM (b), and AFM (c) images of graphene on glass. White arrows point to speckle-like debris. Cyan arrows point to thread-like contaminants likely due to polymer residuals from the wet-transfer process. 

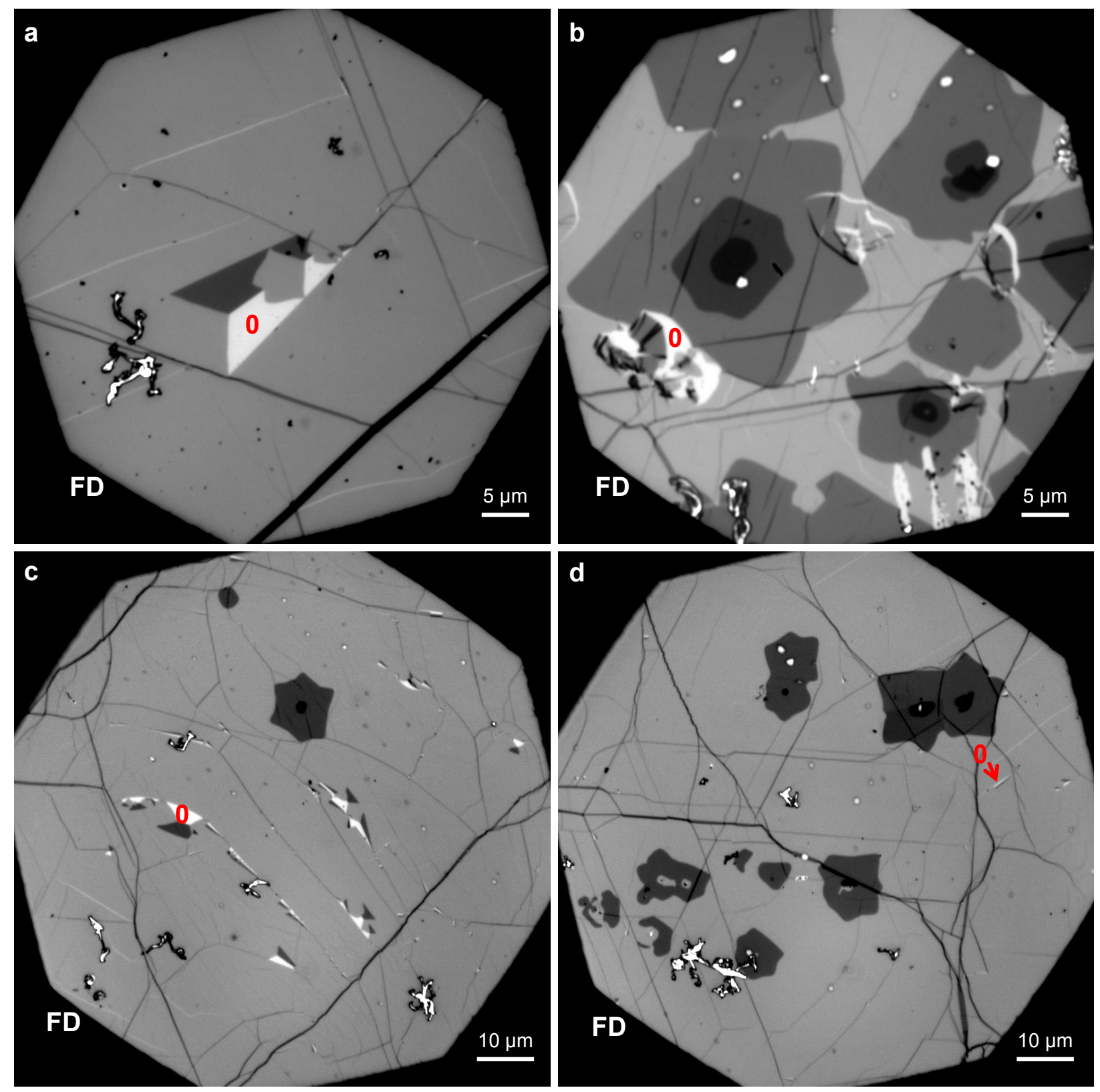

Figure S6. Additional IRM images of graphene on glass substrates. $(a, b)$ Obtained using a 100x oil-immersion objective. (c,d) Obtained using a 60x water-immersion objective. See also Movies S1 and S2 for real-time examination over large areas at 2x and 4x video-rates. FD: field diaphragm of the microscope. " 0 " marks areas with no graphene coverage. 

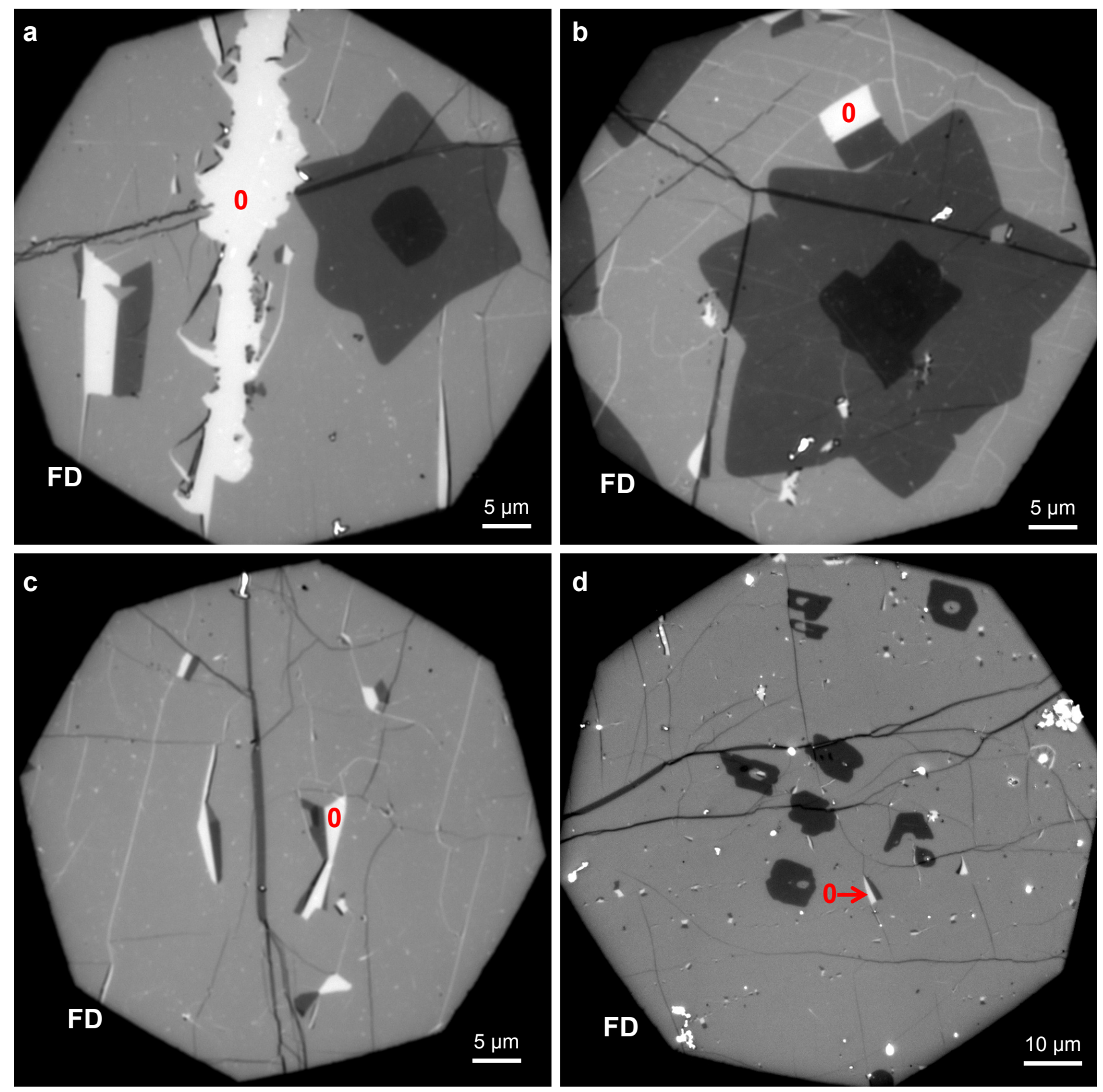

Figure S7. Additional IRM images of graphene on quartz substrates. (a-c) Obtained using a 100x oil-immersion objective. (d) Obtained using a 60x water-immersion objective. FD: field diaphragm of the microscope. " 0 " marks areas with no graphene coverage. 

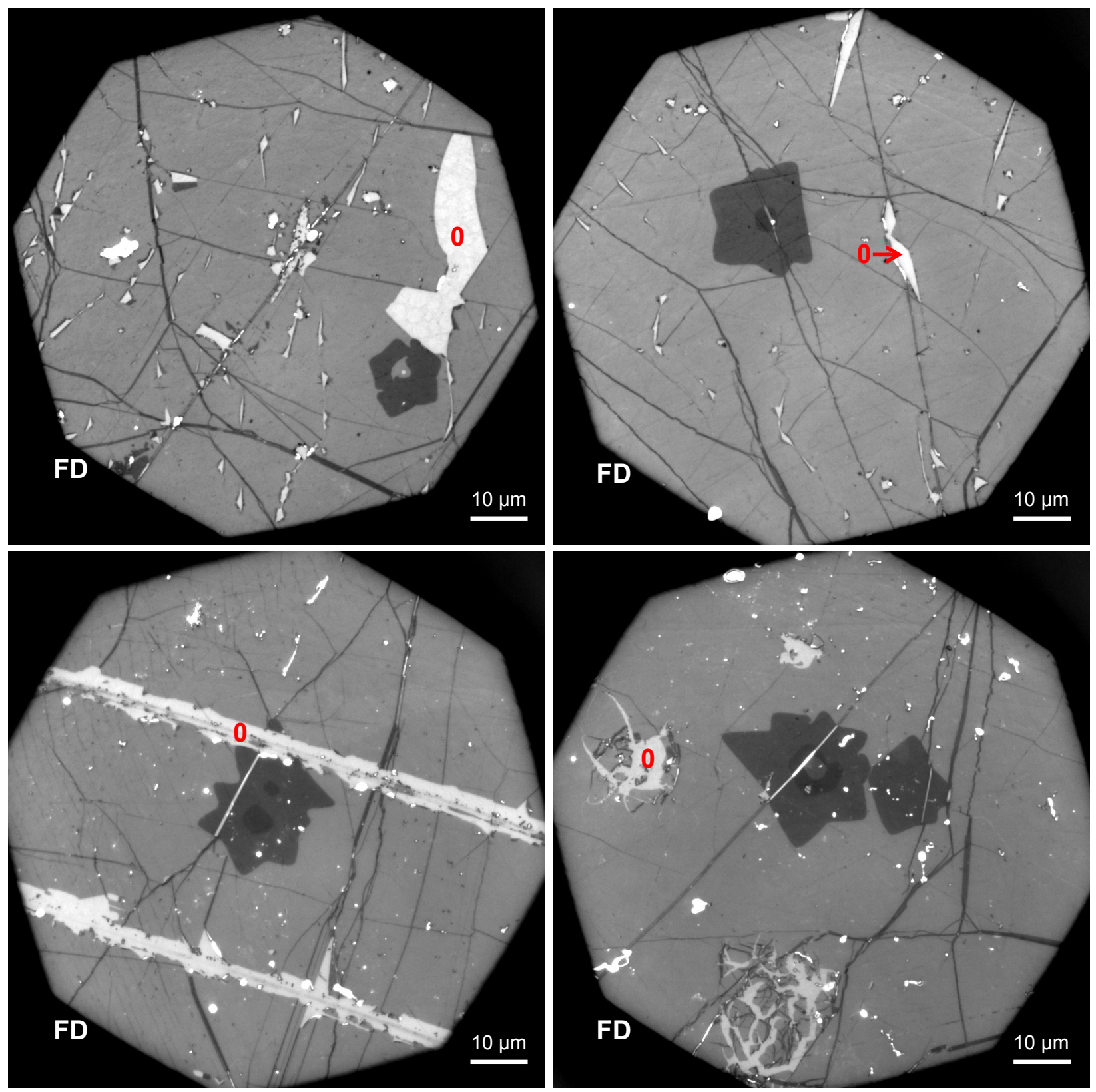

Figure S8. Additional IRM images of graphene on $\mathbf{C a F}_{2}$ substrates. Images were obtained using a 60x water-immersion objective. FD: field diaphragm of the microscope. " 0 " marks areas with no graphene coverage. 

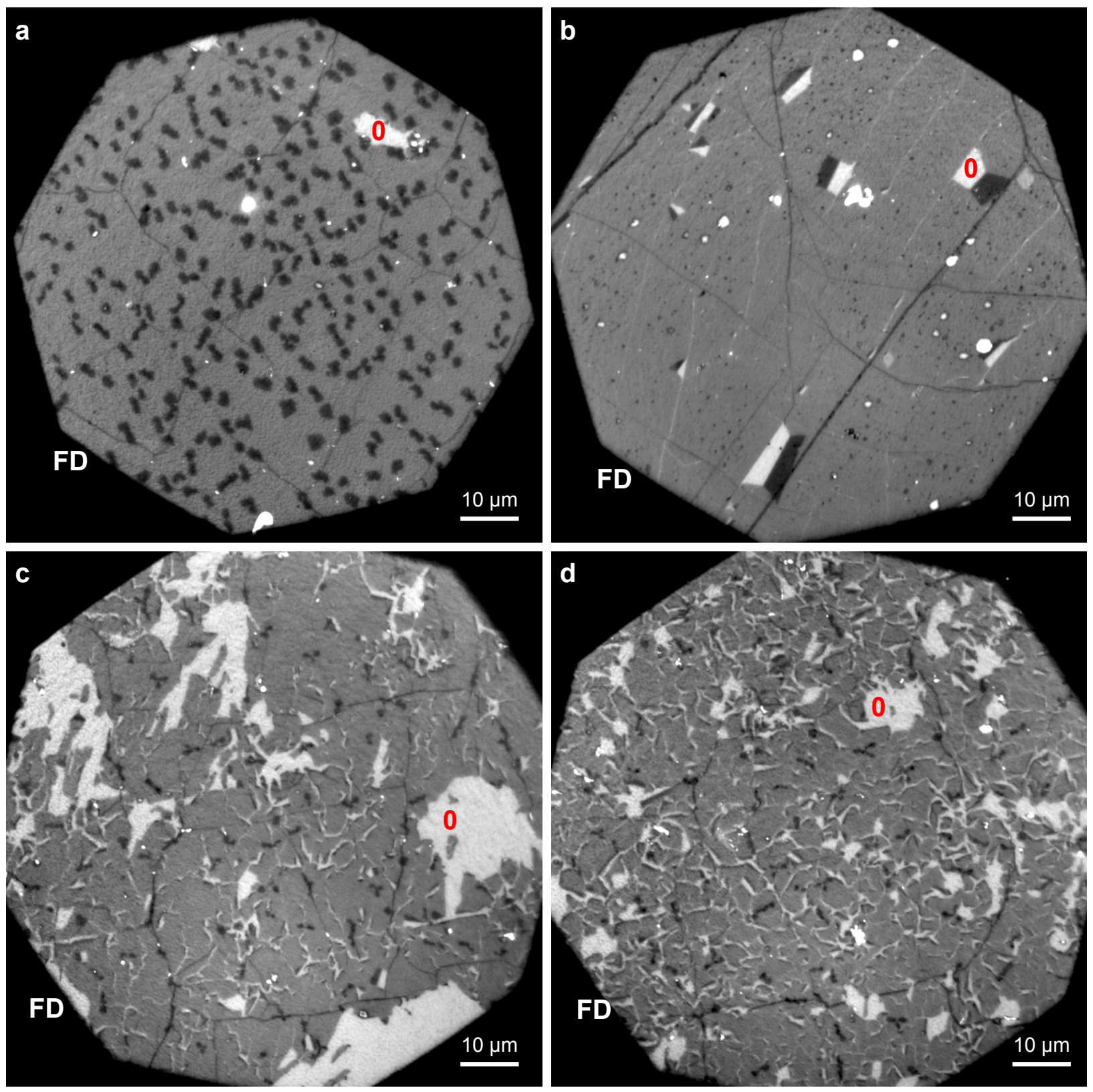

Figure S9. Additional IRM images of graphene on Aclar substrates. (a,b) Graphene transferred using standard PMMA-protected wet-transfer. (c,d) Graphene transferred using thermal release tapes. Imaged were obtained using a 60x water-immersion objective. FD: field diaphragm of the microscope. " 0 " marks areas with no graphene coverage. 

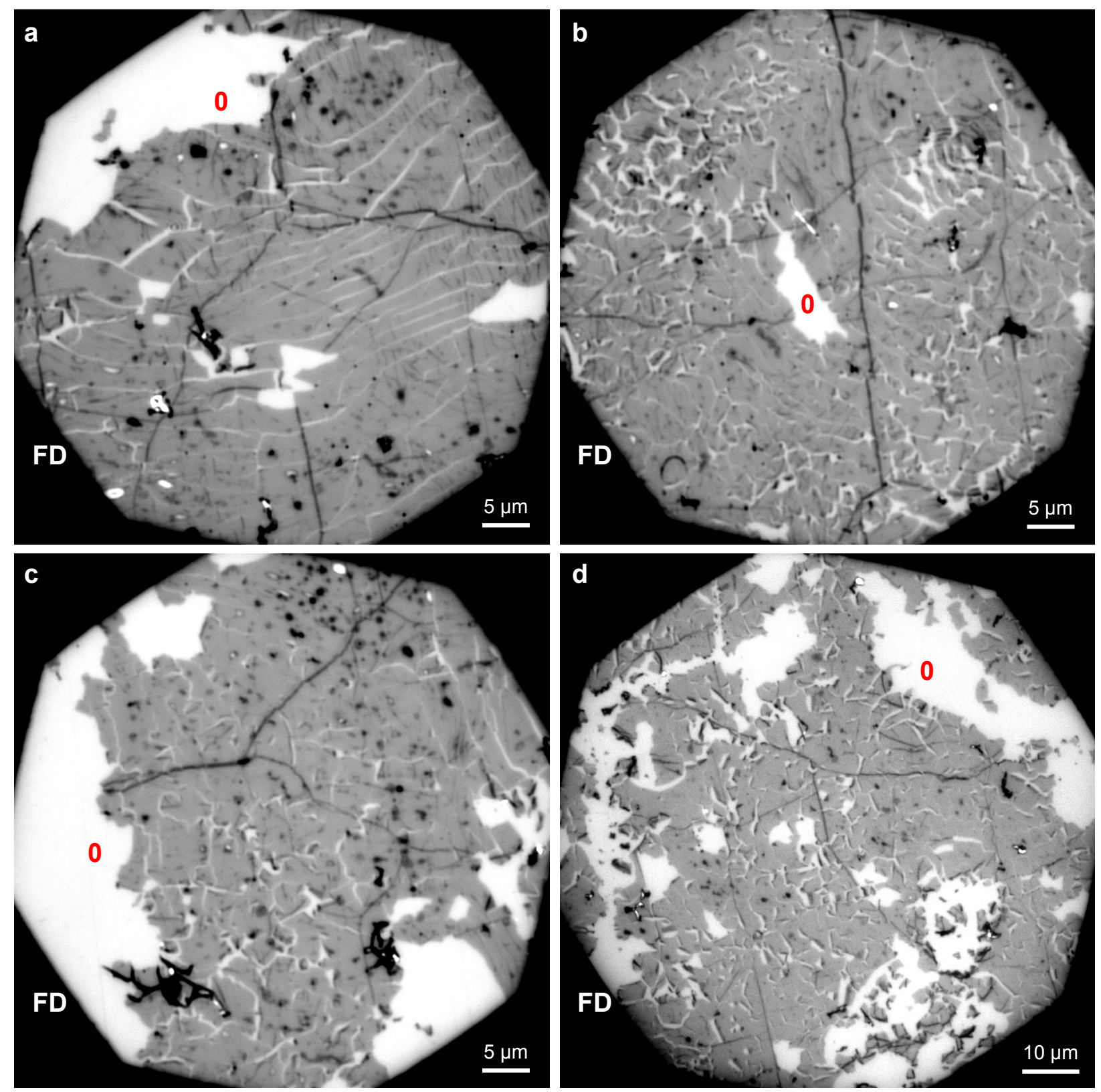

Figure S10. Additional IRM images of graphene on polycarbonate substrates. (a-c) Obtained using a 100x oil-immersion objective. (d) Obtained using a 60x water-immersion objective. Graphene was transferred using thermal release tapes. FD: field diaphragm of the microscope. "0" marks areas with no graphene coverage. 

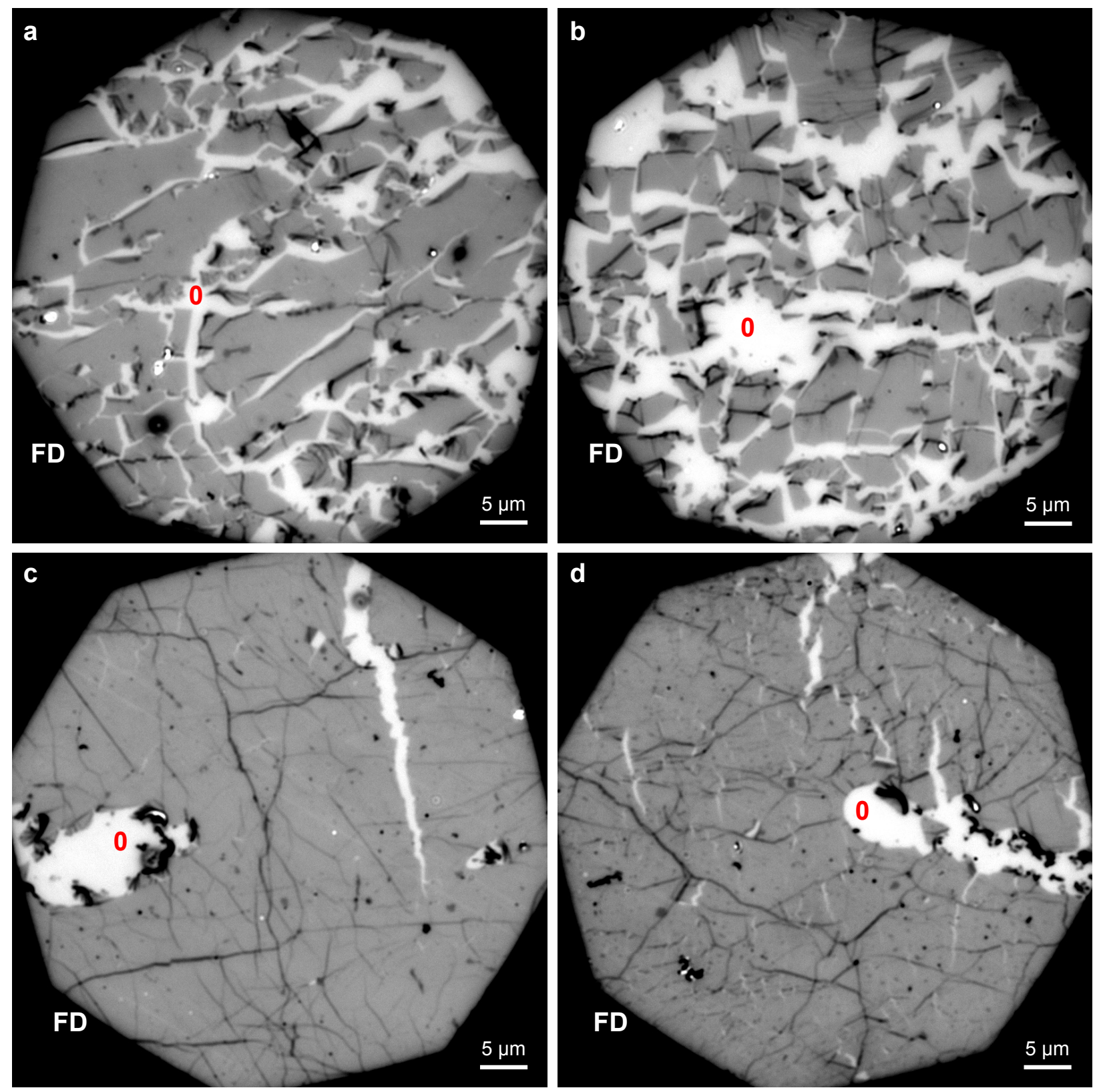

Figure S11. Additional IRM images of graphene on PET substrates. (a,b) Graphene was transferred using Scotch tapes. (c,d) Graphene was transferred using wet transfer without PMMA protection. Images were obtained using a 100x oil-immersion objective. FD: field diaphragm of the microscope. "0" marks areas with no graphene coverage. 

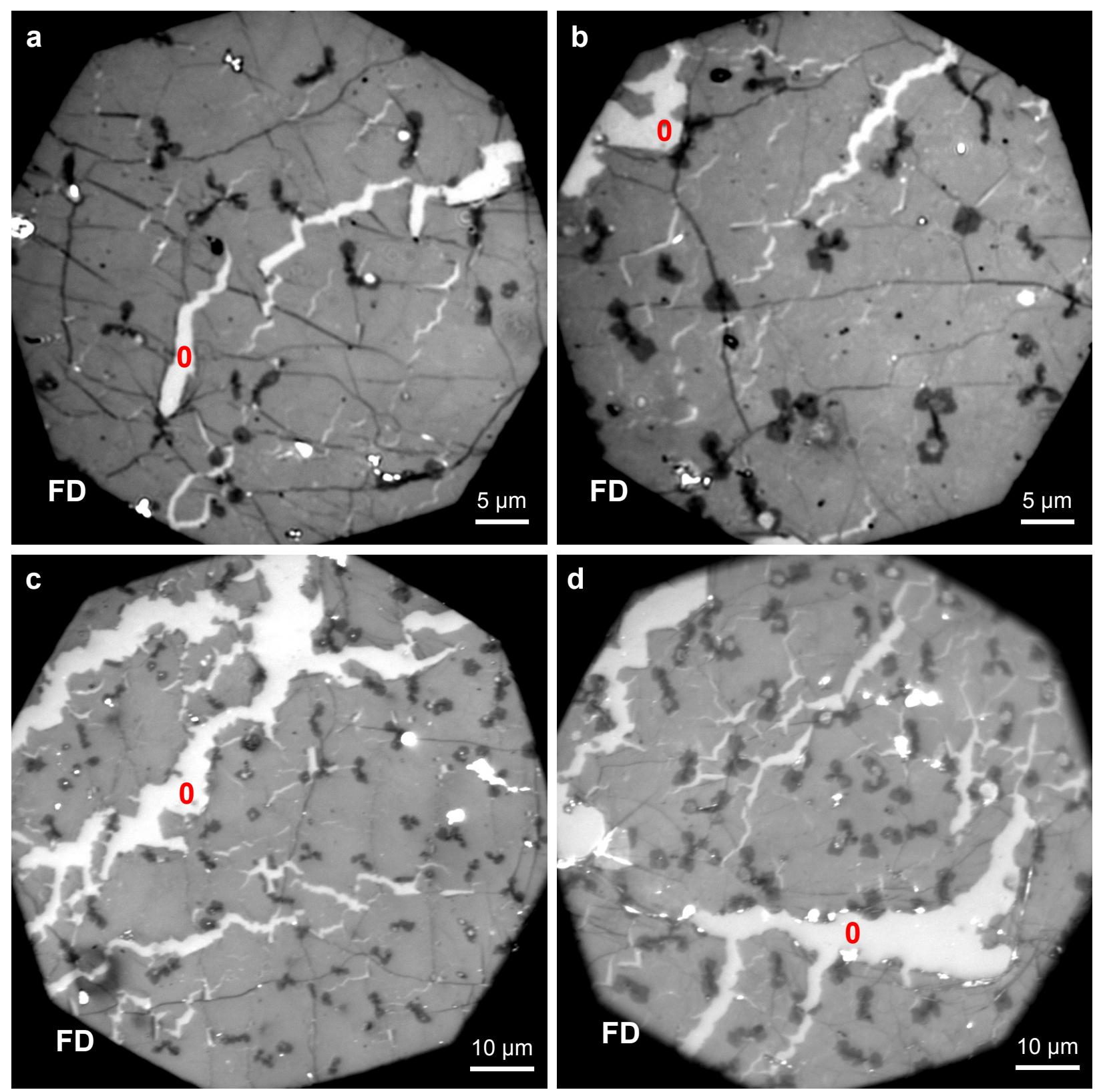

Figure S12. Additional IRM images of graphene on cellulose acetate substrates. $(a, b)$ obtained using an 100x oil-immersion objective. (c,d) Obtained using a 60x water-immersion objective. Graphene was transferred using wet transfer without PMMA protection. FD: field diaphragm of the microscope. "0” marks areas with no graphene coverage. 

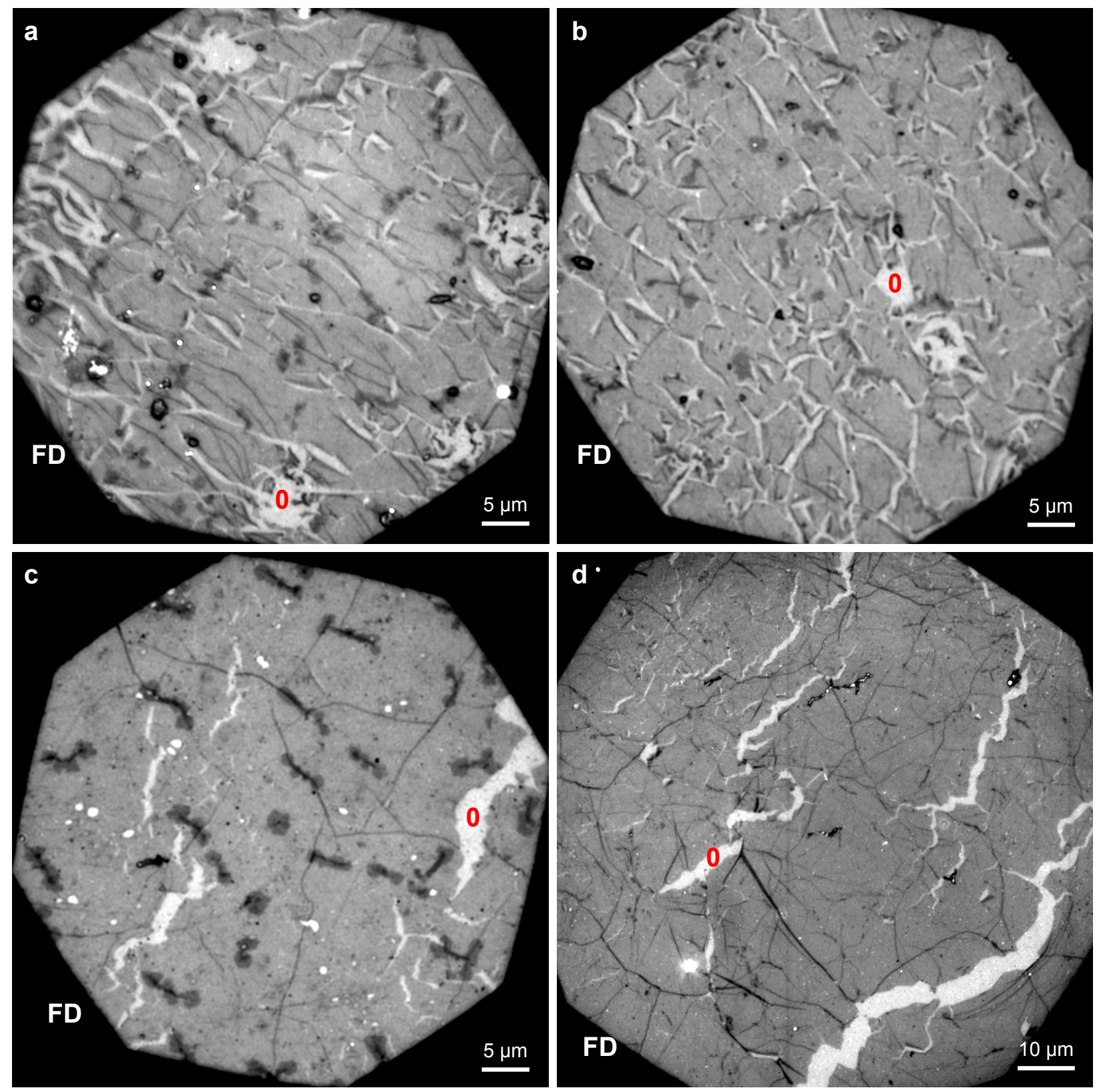

Figure S13. Additional IRM images of graphene on PVC substrates. (a,b) Graphene was transferred using thermal release tapes. (c,d) Graphene was transferred using wet transfer without PMMA protection. Images were obtained using a 100x oil-immersion objective (a-c) or a $60 x$ water-immersion objective (d). FD: field diaphragm of the microscope. " 0 " marks areas with no graphene coverage. 

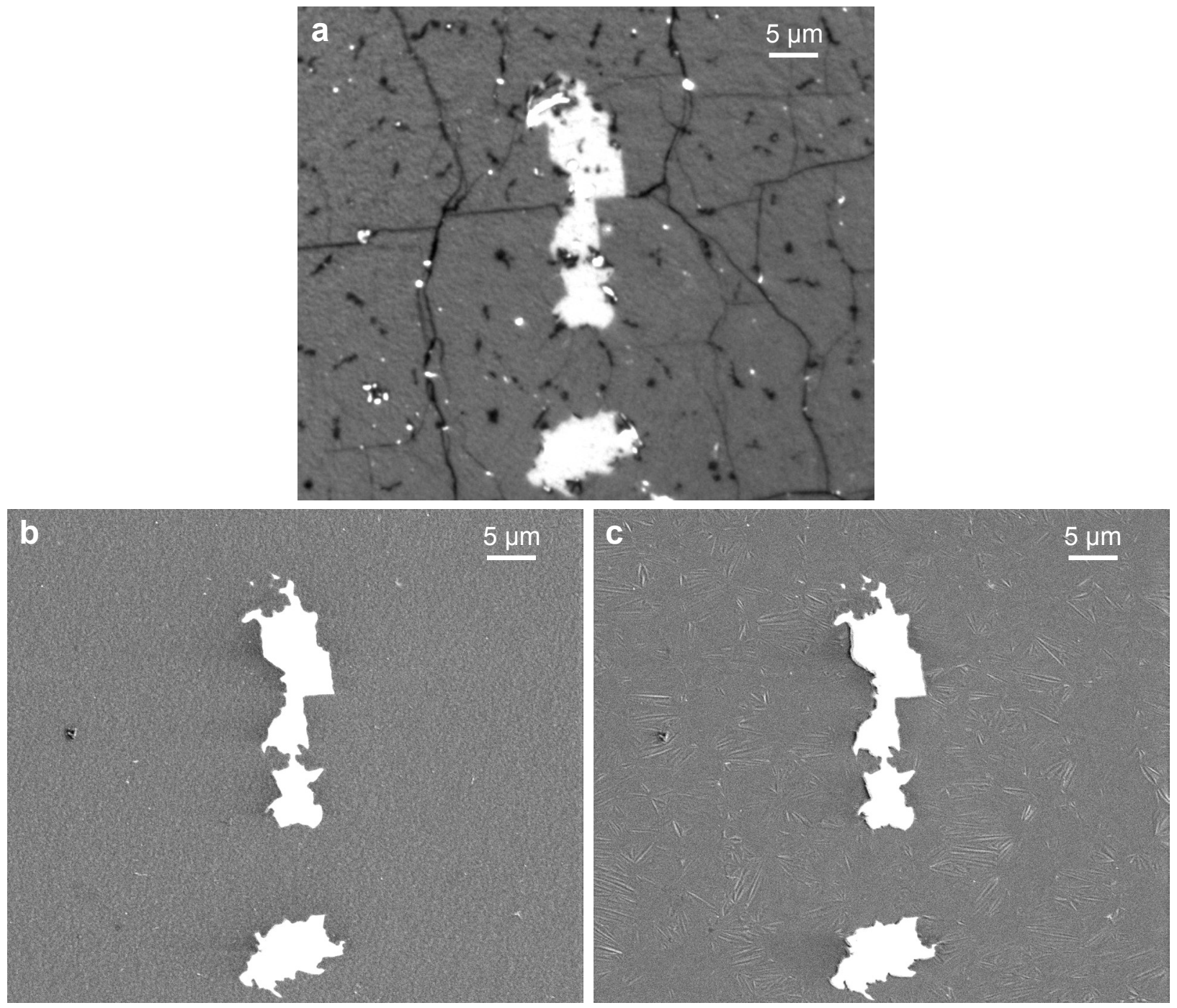

Figure S14. SEM leads to poor contrast and noticeable structural changes for graphene on Aclar. (a) IRM image of graphene on Aclar, showing a monolayer with voids, typical wrinkles, and small islands of bilayers. (b) SEM image of the same area shows a roughened surface; voids are observed due to surface charging, but wrinkles and bilayers are not observed. (c) A subsequent SEM image of the same area taken immediately after (b), showing that significant morphological changes occurred over the entire field, attributable to instability of polymer under electron beam. 
Video S1. Real-time inspection of different areas of graphene on a glass substrate at $2 \times$ video rate (50 frames per second). Playback is set to the standard video frame rate of 25 frames per seconds, and so is slowed-down by 2 -fold when compared to actual time. Note timestamp at upper-left corner. Scale bar: $5 \mu \mathrm{m}$.

Video S2. Real-time inspection of different areas of graphene on a glass substrate at $4 \times$ video rate (100 frames per second). Playback is set to the standard video frame rate of 25 frames per seconds, and so is slowed-down by 4-fold when compared to actual time. Note timestamp at upper-left corner. Scale bar: $5 \mu \mathrm{m}$.

Video S3. In situ IRM monitoring of the microscopic failure mode of graphene on Aclar under uniaxial strain. (a) IRM data. Cyan arrowhead points to a wrinkle that evolves into a crack during stretching. (b) Computer-tracked cracks, with local width coded by color. Strain of each frame is labeled in (b). (c) Strain-dependent crack density and average crack width, calculated from IRM data. (d) Concurrently measured graphene resistance. Scale bars: $5 \mu \mathrm{m}$.

\section{References}

(1) Yeh, P. Optical Waves in Layered Media; Wiley: Hoboken, NJ, 2005.

(2) Reina, A.; Son, H.; Jiao, L.; Fan, B.; Dresselhaus, M. S.; Liu, Z.; Kong, J. J. Phys. Chem. C 2008, 112, 17741-17744.

(3) Li, X. S.; Cai, W. W.; An, J. H.; Kim, S.; Nah, J.; Yang, D. X.; Piner, R.; Velamakanni, A.; Jung, I.; Tutuc, E.; Banerjee, S. K.; Colombo, L.; Ruoff, R. S. Science 2009, 324, 1312-1314.

(4) Wojcik, M.; Hauser, M.; Li, W.; Moon, S.; Xu, K. Nat. Commun. 2015, 6, 7384.

(5) Horcas, I.; Fernandez, R.; Gomez-Rodriguez, J. M.; Colchero, J.; Gomez-Herrero, J.; Baro, A. M. Rev. Sci. Instrum. 2007, 78, 013705.

(6) Curtis, A. S. G. J. Cell Biol. 1964, 20, 199-215.

(7) Verschueren, H. J. Cell Sci. 1985, 75, 279-301.

(8) Limozin, L.; Sengupta, K. ChemPhysChem 2009, 10, 2752-2768.

(9) Pellegrin, S.; Mellor, H. J. Cell Sci. 2007, 120, 3491-3499.

(10) Xu, K.; Babcock, H. P.; Zhuang, X. Nat. Methods 2012, 9, 185-188. 